\title{
The effects of a mindfulness-based program on the incidence of injuries in young male soccer players
}

\author{
Aynollah Naderi \\ Fatemeh Shaabani \\ Hassan Gharayagh Zandi \\ Luis Calmeiro \\ Britton W. Brewer
}

Accepted author manuscript version reprinted, by permission, from Journal of Sport and Exercise Psychology, 2019, https://doi.org/10.1123/jsep.2019-0003 (C) Human Kinetics, Inc.

Naderi, A., Shaabani, F., Zandi, H.G., Calmeiro, L., Brewer, B.W. (2020) 'The effects of a mindfulness-based program on the incidence of injuries in young male soccer players', Journal of Sport and Exercise Psychology. 
1 Running Head: MINDFULNESS TRAINING AND SPORT INJURY PREVENTION

2

3

4

5

6 Aynollah Naderi $^{\mathrm{a},}$ Fatemeh Shaabani ${ }^{\mathrm{b}}$, Hassan Gharayagh Zandi ${ }^{\mathrm{c}}$, Luís Calmeiro ${ }^{\mathrm{d}, \mathrm{e}}$, Britton

$7 \quad$ W.Brewer ${ }^{\mathrm{f}}$

8

$9{ }^{\mathrm{a}}$ School of Sport Sciences, Shahrood University of Technology, Iran; ${ }^{\mathrm{b}}$ Department of Sport

10 Psychology, School of Physical Education and Sport Sciences, Allameh Tabataba'i

11 University, Iran; ${ }^{\mathrm{c}}$ Department of Sport Psychology, School of Physical Education and Sport

12 Sciences, University of Tehran, Iran; ${ }^{\mathrm{d}}$ School of Applied Science, Abertay University,

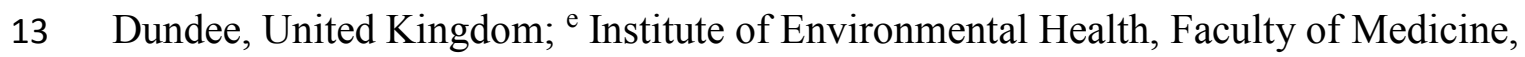

14 University of Lisbon, Portugal; ${ }^{\mathrm{f}}$ Department of Psychology, Springfield College, Springfield, 15 USA.

18 Corresponding author:

19 Luis Calmeiro

20 Abertay University

21 School of Social and Health Sciences

22 Old College 3008

23 Bell Street

24 Dundee DD1 1HG

25 Phone \#: +44 (0)1382 308498

26 1.calmeiro@abertay.ac.uk 


\section{Effectiveness of a Mindfulness-Based Program Aimed at Reducing the Incidence of}

\section{Injuries in Young Male Soccer Players}

Injuries are a common major adverse event during a soccer player's career (Stubbe et al., 2015). It is estimated that $65 \%$ to $95 \%$ of international level male soccer players suffer at least one injury each year (Hägglund, 2007) and the injury incidence ranges from 2 to 19.5 injuries per 1000 hours of soccer practice (Pfirrmann, Herbst, Ingelfinger, Simon, \& Tug, 2016). Sport injuries have high financial costs for the health care system, club, and player (Ekstrand, 2013), and may lead to unsuccessful performance and early retirement from the sporting career (Drew, Raysmith, \& Charlton, 2017). Injured athletes can experience a number of negative psychological and emotional consequences that may include depression, anxiety, sadness, isolation, irritation, lack of motivation, anger, frustration, disordered eating, sleep disturbance, disengagement, or substance use/abuse (Brewer \& Redmond, 2017; Putukian, 2016). Therefore, sport injury prevention strategies are needed with the purpose of decreasing the risk of injury and the negative repercussions that may follow.

To explain the relationship between acute (traumatic) sport injuries and psychological factors, Williams and Andersen (1998) proposed the model of stress and athletic injury. According to this model, a potentially stressful situation (e.g., an important competition) may lead to a stress response, which varies in intensity depending on how the athlete evaluates the situation. This evaluation is known as cognitive appraisal. The more a situation is appraised as threatening, the more intense the athlete's stress response will be (Williams \& Andersen, 1998). The model also considers that personality factors (e.g., trait anxiety, sense of coherence, locus of control, hardiness), history of stressors (e.g., major-life-event stresses, daily hassles, previous injury history), and athlete's coping strategies and available resources (e.g., psychological skill use, social support) may influence the intensity of the stress response and the subsequent likelihood of injury (Rogers \& Landers, 2005; Williams \& 
54 Andersen, 1998). Such increase in athletes' susceptibility to injury is thought to occur due to changes in physiological (e.g., increased muscle tension, fatigue, impaired timing and motor coordination) and attentional (e.g., narrowing of visual field, distractibility, loss task relevant cues) processes (Brewer \& Redmond, 2017). A recent meta-analysis demonstrated that this framework had an acceptable fit to published data and explained $7.3 \%$ of the variance in injury rates (Ivarsson et al., 2017), supporting its relevance for injury prevention.

Until recently, little attention has been paid to psychosocial strategies in injury prevention. In the last 20 years, results of the few psychological intervention studies based on the Williams and Andersen (1998) stress and athletic injury model suggest that interventions directed at the stress response have the potential to decrease the risk of occurrence of acute sport injuries (Ivarsson et al., 2017; Ivarsson, Johnson, \& Edvardsson, 2015; Johnson, Ekengren, \& Andersen, 2005; Perna, Antoni, Baum, Gordon, \& Schneiderman, 2003; Tranaeus, Johnson, Engström, Skillgate, \& Werner, 2015). The majority of these studies, however, have considerable limitations, including lack of attentional control groups (Edvardsson, Ivarsson, \& Johnson, 2012; Perna et al., 2003; Tranaeus et al., 2015) and preferential selection of athletes who are at risk of acute injury (Johnson et al., 2005; Perna et al., 2003; Tranaeus et al., 2015). In injury intervention studies, selecting as participants only athletes who are at risk of suffering an injury is particularly problematic, because doing so can diminish the magnitude of the effects of the interventions and undermine generalizability. In fact, when athletes who are less likely to be injured are included in the sample studies, interventions can be less effective.

Despite the fact that research on mindfulness began in $1960 \mathrm{~s}$, the interest in mindfulness as an acute sport injury prevention strategy is recent (Ivarsson, Johnson, Andersen, Fallby, \& Altemyr, 2015; Ivarsson et al., 2017). Mindfulness is defined as an ability to consciously and intentionally maintain full attention and awareness to the present moment with a non- 
judgmental attitude (Brown \& Ryan, 2003). Different types of mindfulness interventions have been administered in the sport context, but the application of the MindfulnessAcceptance-Commitment approach (MAC; Gardner \& Moore, 2007) is particularly popular in sport. This approach was developed in 2001 based on a combination of key concepts and practice skills adapted from mindfulness-based cognitive therapy (Segal, Teasdale, Williams, \& Gemar, 2002) and acceptance and commitment therapy (Hayes, Strosahl, \& Wilson, 2012). This approach emphasizes the promotion of a non-judging present-moment awareness, the acceptance of one's internal cognitive and affective states, a focused attention on taskrelevant external stimuli, and an effortful values-driven commitment to behaviors that support athletic goals (Gardner \& Moore, 2007). A recent systematic review of 66 studies $(n=3908)$ suggested that MAC approaches may improve performance, but further research is necessary to confirm any causal claims of this relationship (Noetel, Ciarrochi, Van Zanden, \& Lonsdale, 2017).

Studies show that mindfulness practice may affect a variety of psychological processes including improvement of executive functions, attention, and awareness (Brown \& Ryan, 2003), while decreasing anxiety and stress (Goldin \& Gross, 2010). In addition, mindfulness training may attenuate negative appraisals of potentially stressful situations (e.g., an upcoming competition) and facilitate the use of adaptive forms of coping (Weinstein, Brown, \& Ryan, 2009). According to Williams and Andersen (1998), paying attention to the here-and-now (i.e., mindfulness) is part of our evolutionary heritage and it has had tremendous survival value. Complex, distracting stimuli and internal thoughts prevent us from being in the present moment and expose us to harm by thwarting the effective scanning of the environment. Therefore, inappropriate changes in attentional processes such as distractibility and loss of task relevant cues are thought to increase athletes' susceptibility to injury (Brewer \& Redmond, 2017). 
to injury. In a 6-month study with 41 high school Swedish soccer players, Ivarsson, Johnson, Andersen, et al. (2015) reported no statistically significant difference in injury incidence between the mindfulness intervention group and a control group. However, the intervention had a medium effect size (adjusted Cohen's $d=-0.59$ ) on injury incidence, a clinically meaningful outcome: the mindfulness group had almost half the number of injuries of the control group ( 8 vs. 15, respectively). The Ivarsson, Johnson, Andersen, et al. (2015)study had a small sample size that limited generalization of the results. Moreover, they did not assess trait mindfulness at baseline to control for between groups differences. Based on the above, the purpose of our study was two-fold. First, we investigated the extent to which a mindfulness training program could decrease the number of acute sport injuries in young elite soccer players. Second, we explored the role that changes in trait and state mindfulness, trait sport anxiety, stress, and attention control can play in the rate of acute sport injuries. Hence, the current study expanded on that of Ivarsson, Johnson, Andersen, et al. (2015) by considering a number of psychological variables that may mediate the effects of mindfulness training on injury susceptibility. Furthermore, we also controlled for athlete-exposure as it changes the probability of the occurrence of injuries. We hypothesized that mindfulness practice would reduce trait sport anxiety and stress, improve attentional processes, and reduce acute sport injury occurrence. In addition, we expected that the level of sport anxiety and stress in injured athletes would be higher than those of the uninjured athletes. We also expected that the levels of attention and state and trait mindfulness would be lower in injured athletes than in uninjured athletes. Finally, using a mediation model, we hypothesized that trait and state mindfulness, perceived stress, trait sport anxiety, and attentional process would mediate the association between mindfulness training and sport injury. 


\section{Method}

130

131

132

133

134

135

136

137

\section{Study Design}

Prospective study conducted during one season from July 2016 to May 2017 in

(Blind).

\section{Participants}

One hundred sixty-eight junior soccer players aged 16 to 19 years old (mean age $=$ $17.1 \pm 1.1$ years) participated in the study and were recruited from eight clubs certified by the (Blind). The clubs were selected by the local soccer association. Of the participants, 21\% (two clubs) played at the highest junior level and the remaining 79\% (six clubs) competed at the senior elite level in (Blind). Teams had at least two regular soccer training sessions and one match per week. Athletes participated in soccer related activities for 8 to 12 hours per week.

To be eligible to participate in the study, athletes had to attend at least $90 \%$ of their practice sessions and provide information about their injuries. After completion of the baseline measures and obtaining all medical clearances, eligible participants were randomly allocated into mindfulness $(\mathrm{n}=84$; age $=17.44 \pm 1.12$ years; height $=171 \pm 5.78 \mathrm{~cm}$; $\mathrm{BMI}=$ $19.1 \pm 1.47)$ or control $(\mathrm{n}=84$; age $=17.6 \pm 1.23$ years; height $=172 \pm 5.74 \mathrm{~cm} ; \mathrm{BMI}=$ $18.68 \pm 1.43)$ groups.

Three participants from the intervention group and five participants from the control group did not complete the minimum of sessions required, therefore they were excluded from the study and their data were not considered for further analysis. The final sample consisted of 160 athletes, 79 in the control group and 81 in the intervention group.

The sample size was calculated assuming an injury incidence of $63 \%$ reported in a previous study with 16 to 19 year-old males (Stubbe et al., 2015). An a priori sample size 
calculation estimated that 168 participants (i.e., 84 per group) were required to provide $80 \%$ power to detect a $50 \%$ effect size in the intervention group (alpha set at $5 \%$ ).

\section{Data Collection}

Injury recording. An injury was defined as any physical complaint incurred during a competition or training directly related to soccer that resulted in four or more days of absence from practice, including the day the injury was reported. The player was considered injured until deemed apt to return to training and competition by the medical staff. The severity of the injury was classified according to the duration of complaints and absence from matches and training sessions: mild, when there was an absence of 4-7 days or complaints for more than 2 weeks; moderate, when there was an absence for 8-28 days; and severe, when there was an absence of more than 28 days (Fuller et al., 2006). If the injury was caused by repetitive microtrauma without a distinguishable traumatic event, it was considered an overuse injury and was excluded from our study.

Comprehensive Inventory of Mindfulness Experiences (CHIME; Bergomi, Tschacher, \& Kupper, 2014). CHIME is a 37-item inventory that assesses trait mindfulness. The inventory describes various behaviors or experiences participants may have had during the previous two weeks. Participants were asked to rank their mindful engagement on a six-point Likert scale, ranging from 1 (almost never) to 6 (almost always) in each of those scenarios. Bergomi et al. (2014) supported the reliability (internal consistency and retest-reliability) and validity (construct, criterion, and incremental) of the CHIME and reported satisfactory psychometric properties.

Toronto Mindfulness Scale (TMS; Lau et al., 2006). TMS is a state mindfulness measure that captures the extent to which participants experienced a feeling of heightened awareness, as well as the quality of such awareness. It includes 13 items on 5-point Likert 
scales, ranging from 0 (not at all) to 4 (very much). Higher total scores indicate higher overall state mindfulness. Reliability and validity of TMS were supported by Lau et al. (2006).

Sport Anxiety Scale-2 (SAS-2; Smith, Smoll, Cumming, \& Grossbard, 2006). This scale consists of 21 items that measure the competitive trait anxiety experienced by athletes before or during competition. SAS-2 includes three subscales comprised of four items each: worry (e.g., "I worry that I will not play well"), somatic anxiety (e.g., "My body feels tense"), and concentration disruption (e.g., "It is hard to concentrate"). Participants were asked to indicate on 4-point Likert scales, ranging from 1 (not at all) to 4 (very much), how they generally feel before or during sporting competitions. An overall trait anxiety score is calculated as the sum of all items and varies from 21 to 84, with high scores indicating high trait anxiety. Smith et al. (2006) obtained support for the reliability and validity of the SAS-2, indicating sound psychometric properties.

Kessler Psychological Distress Scale (K10;Andrews \& Slade, 2001). K10 is a 10-item questionnaire designed to measure different types of psychological stress responses participants might have experienced during the last 30 days, such as "feeling tired out for no good reason" or "sad or depressed." Responses are scored on 5-point Likert scales ranging from 0 (none of the time) to 4 (all of the time). The total score varies between 0 and 40, with higher scores indicating higher levels of psychological distress. The K10 is a valid and reliable measure of psychological distress and its psychometric properties are consistent across a variety of sociodemographic subsamples (Andrews \& Slade, 2001; Kessler et al., 2002).

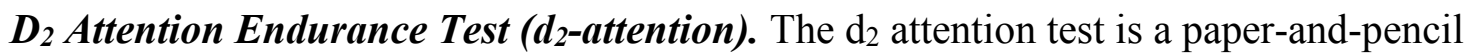
version test used to determine the capacity to focus on one stimulus, while suppressing awareness of competing stimuli. Participants were asked to cross out any letter "d" with two marks above it or below it in any order, while ignoring distractors, which are similar to the 
target stimulus (e.g., "p" with two marks or a "d" with one or three marks). This test consists of 14 rows with 47 randomly presented " $p$ " and " $d$ " letters in each line. Participants' scores were determined by the total number of letters processed (TN), total number of letters correctly marked (CP), and percentage of errors (errors of omission and commission) (E \%).

TN was an indicator of processing speed and amount of work completed, CP was an indicator of concentration, and $\mathrm{E} \%$ was an indicator of quality of attention (Bates \& Lemay, 2004). In a series of test-retests, $\mathrm{d}_{2}$ test indices TN, TN-E, and CP demonstrated satisfactory to good reliability $(r>.70)$. In our study, we used the average of $\mathrm{z}$ scores of the $\mathrm{d} 2$ attention test as a measure of attention. We calculated the $\mathrm{d} 2$ attention test score as follows: Total score of attention test $=\left(\mathrm{z}\right.$ score of $\mathrm{d}_{2}-\mathrm{TN}+\mathrm{z}$ score of $\mathrm{d} 2-\mathrm{CP}+\mathrm{z}$ score $\left.\mathrm{d}_{2}-\mathrm{E} \%\right) / 3$. In previous research, internal consistency coefficients (Cronbach's alpha) ranging from .93 to .96 and reliability coefficient (ICC) from.69 to .89 for athlete participants were observed (Caglar \& Koruc, 2006). In addition, Barkley (1991) reported that paper-and-pencil tests, rather than computer-administered versions, have resulted in higher correlations between children's test scores and parent and teacher ratings of attention.

\section{Athletes' and Coaches' Intervention Satisfaction Questionnaire}

Coaches and athletes were asked to respond to a researcher-administered questionnaire based on 7-point Likert scales. Coaches' questions included: (a) to what extent did the mindfulness program interrupt your training plan? $(1=$ never to $7=$ always $)$, (b) how satisfied are you with the effects of the mindfulness program on your soccer players' performance? $(1=$ extremely dissatisfied to $7=$ extremely satisfied $)$, and $(\mathrm{c})$ how willing are you to use such interventions in the future? $(1=$ extremely unwilling to $7=$ extremely willing).

Athletes' answered the following questions: (a) how do you rate your level of satisfaction with the mindfulness program delivery? $(1=$ extremely dissatisfied to $7=$ 
extremely satisfied); (b) how do you rate your level of understanding of the information provided in the sessions $(1=$ poor to $7=$ excellent $)$; $(\mathrm{c})$ how do you rate the quality of the communication with the psychologist $(1=$ poor to $7=$ excellent $)$; $(\mathrm{d})$ how do you rate your level of improvement of the skills taught $(1=$ poor to $7=$ excellent $)$; (e) how well are you able to apply the skills learned in training and competition $(1=$ poor to $7=$ excellent $)$, and $(\mathrm{f})$ to what extent did the mindfulness program improve your soccer performance $(1=$ poorly to $7=$ excellent $)$

\section{Mindfulness Intervention Program}

The mindfulness intervention was developed according to Gardner and Moore's (2007) MAC approach and its contents are illustrated in supplementary file 1 (Gardner \& Moore, 2007; Ivarsson, Johnson, \& Edvardsson, 2015; Plemmons, 2015). The mindfulness intervention consisted of seven sessions, one 45-minute session per week. All sessions started with a shared discussion among participants about their experiences, feelings, and thoughts concerning the previous session, followed by the instructor's personalized feedback to each athlete. This feedback pertained to information provided during the shared discussion and helped develop or reinforce an understanding of the content presented in the previous meeting. Each session included a specific topic in line with the MAC manual (Gardner \& Moore, 2007), followed by a presentation concerning the mechanisms of the MAC approach and participants' discussions. All sessions ended with mindfulness exercises, specific to the session topic. At the end of each session, an audio mindfulness exercise was sent electronically to the participants' mobile phones and/or e-mail addresses. Participants were required to listen to the audio to complement their training.

\section{Attention Control Intervention Program}

In the attention control group (CG), participants attended seven sessions of presentations about the psychology of sport injury. As in the mindfulness group, participants 
in the control group met once weekly for 45 minutes. Contents for this group were developed based on a book on the psychology of sport injury and it included the topics illustrated in supplementary file 2 (Brewer \& Redmond, 2017).

\section{Procedure}

This study was reviewed and approved by the Institution Review Board of (Blinded) and all procedures were in accordance with the Declaration of Helsinki. In order to access and speak to potential participants, we contacted team coaches and set a time and place for a meeting. At this meeting, coaches allowed us to inform the players and their parents about the study. Then, participants, coaches, and parents of participants who were under 18 years of age signed an informed consent form.

First, all players completed a face-to-face guided interview history form to collect information regarding participants' demographic information and previous acute sport injury history (i.e., the number of injuries suffered in the previous season of 2015-2016). The injury information allowed participants to be matched and allocated to either the control or the experimental groups. In addition, groups were matched based on team's competition level. None of the teams had a physician, physiotherapist, or psychologist. Then, the Comprehensive Inventory of Mindfulness Experiences (CHIME) was administered to control for the effect of trait mindfulness on stress, anxiety, and rate of injury. Moreover, the Toronto Mindfulness Scale (TMS), Sport Anxiety Scale-2 (SAS-2), and Kessler Psychological Distress Scale (K10) were administered before the intervention (pretest) and monthly, for five months after the end of the intervention, to assess changes in the state mindfulness, trait anxiety, and stress during the season. The post-test measurements used in the analysis were taken immediately before the injury had occurred. The use of the last score before the injury avoids the confounding negative effect of the injury on the 
psychological constructs studied. All psychological questionnaires were administered by a trained researcher.

Prior to initiating the mindfulness intervention sessions, participants of both groups were divided into smaller groups containing 15 or 16 participants to maximize active participation, opportunities to receive feedback and acquisition and transfer of information. To facilitate interaction during the sessions, intervention leaders led group discussions using experiential learning techniques and encouraged participants to share their experiences and opinions. All sessions were conducted at the clubs' facilities from April to May 2016. A Ph.D. clinical psychology student and an MSc sport psychology student delivered both programs. During the seven weeks, participants were required to incorporate mindfulness into their daily activities and to practice at least 10 minutes daily. Participants recorded their daily practice in a logbook and all of them reported practicing at least 30 minutes per day.

After the initial data collection, players were followed throughout the season (July 2016 to May 2017) in practice and competition. One physical therapist and one medical student recorded all injuries that met our operational definitions. When an injury was reported, the player or the coach was contacted by the physiotherapist or the medical student to complete a standardized interview on the circumstances of the injury. To maintain updated injury records, at the end of each week, all athletes answered questions concerning match exposure, training exposure and time-loss injuries. All injuries were described in relation to their type and location, date of injury occurrence, date of return to full participation in practice, and the duration of subsequent limitation in match participation.

Athlete-exposure was operationally defined as the athlete's participation in scheduled team practices or competitions during the course of the season. In each session, coaches quantitatively recorded athlete-exposure, and whether or not the warm-up had been conducted and the athletes who had participated in it. Coaches recorded this information on a 
weekly registration form and sent it by e-mail to the research coordinator. Therefore, athleteexposure represents a unit of susceptibility to injury during which the athlete is exposed to the possibility of injury. The more the athlete is exposed to the possibility of injury, the more likely the athletic injury will occur. For this reason, the amount of athlete-exposure was controlled in both groups, allowing for between-group comparisons of the frequency of sport injury. In other words, we ensured that group differences in injury were not due to differences in athlete-exposure.

\section{Data Analyses}

All statistical analyses were conducted using SPSS statistical software (Version 18.0, SPSS Inc., Chicago, IL). Injury incidence was calculated by dividing the number of injuries by the total number of athlete-exposures and reported as injuries per 1000 athlete-exposures. Descriptive statistics (frequencies, means, and standard deviations) were calculated for all variables. Independent $t$-tests were used to compare demographic data between the control group and mindfulness group for pretest, posttest and pretest posttest different. $\chi^{2}$ tests were used to compare the frequency of injuries between groups. Mixed repeated-measures ANOVAs were used to evaluate the within- and between-subject differences in psychological factors between the mindfulness and attention control groups. Additionally, we used an independent $t$-test to compare the average change of psychological factors from pretest to posttest between injured and non-injured participants.

To better understand the magnitude of training gains in the independent groups $t$-test, Cohen's $d_{z}$, expressing the effect size of the between-groups differences, were calculated with values of $\leq .19, .20-.49, .50-.80$, and $\geq .81$ representing trivial, small, medium, and large effects, respectively. Additionally, partial $\eta^{2}$ was reported as an effect size for the repeatedmeasures ANOVA, with .01, .09, and .25 corresponding to small, medium, and large effects. 
A mediation model was used to assess the effects of the mindfulness intervention on sport injury occurrence and the potential indirect effects of psychological factors on this relationship. The SPSS macro PROCESS ( Hayes, 2013) was used to implement a parallel mediation model. Following standard procedures described by Hayes (2013), we formally tested whether the indirect or mediated effect - the difference between the total effect (path c) and the direct effect (path c') - was different from zero using the bootstrapping method $(5,000$ bootstrap samples) and $95 \%$ confidence intervals (CIs). For these analyses, the outcome variable was sport injury (injured or non-injured), the predictor variable was group classification (mindfulness training or control group), and the mediator variables were state mindfulness, trait anxiety, perceived stress, and attention.

The alpha level was set at $\mathrm{p}<.05$, but for multiple comparisons we applied the Bonferroni adjustment and established statistical significance at $\mathrm{p}<.01$ by dividing the common probability level of .05 by 5 (i.e., the number of psychosocial variables).

\section{Results}

Results of the intervention satisfaction interview showed that coaches did not consider that the mindfulness program interfered with their training plan $(3.76 \pm .84$,out of 7$)$ and they were very satisfied with the effect of the intervention on players' performance $(6.24 \pm$ 1.13 ,out of 7). Coaches reported that they were willing to promote such a program in the future $(6.12 \pm 1.02)$. Regarding soccer players' responses, the mean scores for satisfaction with the program (6.14 \pm 1.13$)$, ability to understand sessions (5.76 \pm 1.23$)$, quality of communication with the psychologist $(6.39 \pm 1.12)$, perceptions of their improvement in the skills taught $(5.87 \pm 1.13)$, and generalization to training and competition $(6.14 \pm 1.12)$ were highly favorable.

A series of mixed repeated-measures ANOVAs were conducted to compare trait and state mindfulness, stress, trait anxiety, and attention scores between groups. The main effects 
of time were significant for all psychological variables and subscales $(p \leq .05)$. Regarding the main effects of group, there were no significant group differences for psychological variables $(p>.05)$ except for the trait sport anxiety $(\mathrm{p} \leq .05)$. Findings revealed a significant time $\times$ group interaction for state mindfulness, $F(1,158)=9.36, p=.003, \eta^{2} \mathrm{p}=.05$, and trait mindfulness, $F(1,158)=7.52, p=.007, \eta_{\mathrm{p}}^{2}=.04$, indicating higher state and trait mindfulness after the intervention for the mindfulness group compared to the control group. There was also a significant time $\times$ group interaction for the attention score, $F(1,158)=$ $11.52, p=.001, \eta_{\mathrm{p}}^{2}=.07$, indicating better attentional processes after the interventions for the mindfulness group compared with the control group. There was also a significant time $\times$ group interaction for stress, $F(1,158)=14.34, p=.001, \eta_{\mathrm{p}}^{2}=.08$, and trait sport anxiety, $F$ $(1,158)=15.23, p=.001, \eta_{p}^{2}=.08$, indicating, lower levels of stress and trait anxiety after the intervention for the mindfulness group compared with the control group. The range of the partial $\eta^{2}$ effect size was from .04 to .08 , indicating that mindfulness training had small to medium effects on the psychological variables for the participants in the mindfulness group. Descriptive statics and repeated-measures ANOVA results are displayed in Table 1.

\section{INSERT Table 1 ABOUT HERE}

The total (5,942 units vs. 5,887 units), game (1,351 vs. 1,403), and practice (4,591 vs. $4,485)$ athlete-exposure for MG and CG, respectively, were similar $(p>.05)$. The absolute frequency of injuries in the MG was significantly lower than the absolute frequency of injuries in the CG (22 vs. $\left.36 ; \chi^{2}=5.09, p=.02\right)$. In addition, the number (218 vs. 516$)$ and mean $(9.88 \pm 8.56$ vs. $14.32 \pm 12.28)$ of days lost to injury in the MG was significantly lower than those in the CG, $t(1,158)=3.01, p=.03$. Injury severity was not significantly different between the MG and the CG, $\chi^{2}=1.34, \mathrm{p}=.47$, while the incidence of injury per 1000 
athlete-exposures was significantly lower in the $\mathrm{MG}$ than in the $\mathrm{CG}, t(1,158)=2.76, p=.03$. This indicates that MG athletes experienced fewer acute sport injuries than MG athletes in the same amount of soccer participation time (1000 athlete-exposures; Table 2).

\section{INSERT Table 2 ABOUT HERE}

For the mediation analysis, we followed the four-step procedure to test the mediation effect and estimated psychological factors for the mediation effect with PROCESS macro by Hayes (2013). This model included mindfulness training as the antecedent variable (mindfulness intervention group $=1$, control group $=0$ ), injury as the outcome variable (injury $=1$; non-injury $=0$ ), and pretest-posttest changes in state mindfulness, attention, trait anxiety, and perceived stress as mediators. In addition, we used pre-test scores of state mindfulness, attention, trait anxiety, and perceived stress and number of athlete-exposures as covariates. Results show that mindfulness training was negatively related to trait sport anxiety $(b=-3.36, p<.001)$ and stress $(b=-1.41, p<.001)$, and positively related to attention $(b=.57, p<.001)$, state mindfulness $(b=2.37, p<.001)$, and trait mindfulness $(b=$ $3.40, p<.01)$. When the model controlled for mindfulness training, all psychological variables were significantly related to sport injury $(\mathrm{p}<0.05)$ except trait mindfulness $(b=$ $.10, p=.06)$ and stress $(b=-.41, p=.07)$.

The final model showed a significant total effect of mindfulness training on acute sport injury, model LL $=157.82, p<.001$, explaining $72 \%$ of the variance. The direct effect of mindfulness training on sport injury was significant, 2.61, bootstrap $\mathrm{SE}=0.98(95 \% \mathrm{CI}$ : $0.68,4.53)$. In addition, a bias-corrected bootstrap test indicated a significant total indirect effect of mindfulness training on injury through the psychological variables, -5.10 (95\% CI: $806.69,-4.13)$. Considering direct and indirect effects have opposite signs and the direct 
400 effect is larger than the total effect, that's an inconsistent mediation model and it provides

401 evidence of suppression effects. We identified the attention through additional analysis as

402 suppressor variable that removing it from the mediation model, the suppression effect

403 disappears $($ direct effect $=1.03$, non-significant; indirect effect $=-1.70$; and the total effect $=$

$404-0.67)$. Specific indirect effects of mindfulness training on acute sport injury through state

405 mindfulness, -.56 (95\% CI: -73.01, -0.05), attention, -2.17 (95\% CI: -3374.06, -1.42), and

406 trait sport anxiety, -1.41 (95\% CI: -200.04, -0.87) were statistically significant. Considering

407 the percentage of the total indirect effect of the mindfulness training on sport injury

408 accounted for by the indirect effect of each mediating variable, the indirect effects

409 represented $10.98 \%$ for state mindfulness, $27.64 \%$ for trait sport anxiety, and $42.54 \%$ for

410 attention, of the corresponding total effects. The remaining $18.84 \%$ of the indirect effects of

411 the mindfulness training on sport injury related to trait mindfulness and stress. Finally, the

412 indirect effect of attention was significantly higher than the indirect effect of state

413 mindfulness, 1.08, bootstrap SE $=.79(95 \% \mathrm{CI}:-.18,3.17)$.

INSERT Fig 1 ABOUT HERE intervention could decrease the number of acute sport injuries in young soccer players. The main finding of the present study is that mindfulness-based training may be an effective strategy to reduce the incidence of acute sport injuries in young soccer players during one season. More specifically, the intervention reduced the number of injuries and the mean time lost to injury during the season. In addition, mindfulness training reduced anxiety and stress, and improved attention and state mindfulness in young soccer players. These changes were 
small to medium in magnitude $\left(.04<\eta^{2}<.08\right)$. In addition, the mediation model showed that changes in psychological variables such as state mindfulness, attention, stress, and trait sport anxiety were significant mediators of the effects of mindfulness training on the risk of acute sport injury. Our results show that mindfulness training is twice more likely to reduce the risk of acute sport injury than no training through the positive effects of mindfulness on psychological variables.

In our study, the total effect of mindfulness training on acute sport injuries was -2.49 and the indirect effect was -5.10 . However, the direct effect was positive with a magnitude of 2.61 in the unanticipated direction. That is, direct and indirect effects have opposite signs and the direct effect is larger than the total effect. This is known as an inconsistent mediation model and it provides evidence of suppression (MacKinnon, Krull \& Lockwood, 2000). That is, a third variable is influencing the magnitude of the relationship between the dependent and the independent variables resulting in a pathway in which mindfulness training is associated with an increase in acute sport injuries. Therefore, we tested a variety of models, alternately removing each mediator from the analysis, and observed that removing attention and anxiety from the mediation model, the suppression effect disappears (direct effect $=1.03$, nonsignificant; indirect effect $=-1.70$ and the total effect $=-0.67)$. As this suppression effect of attention is likely to be a statistical artifact, and attention is theoretically meaningful as a mechanism through which mindfulness affect injury rates along with other mediators, we proceed with the discussion of the indirect effects.

Nevertheless, surprisingly, the direct effects of the mindfulness intervention in sport injury are contrary to what was expected. A positive relationship between mindfulness training and injury was observed; however, the total effects were according to expectations, so that mindfulness training positively influenced attention and state mindfulness and negatively influenced trait anxiety and perceived stress, which in turn was associated with 
decreased sport injury. These findings are consistent with the results of studies that investigated the effects of psychological skills training (Tranaeus et al., 2015), cognitive behavioral therapy (Edvardsson et al., 2012), and mindfulness (Ivarsson, Johnson, Andersen, et al., 2015) in the reduction of acute sport injury in soccer players. Although the interventions may vary slightly in terms of implementation, their goals are often similar: stress management, emotion control, and attention regulation. Therefore, given that the mindfulness intervention has a role in stress management, emotion control, and attention regulation, we suggest that some processes through which mindfulness may help reduce injury are improved attention and a decreased tendency to perceive situations in stressinducing ways.

Several potential explanations can be proposed to justify the lower injury rate reported in the mindfulness group compared to the control group. First, the present study shows that mindfulness training seems to have decreased the susceptibility to injury through improvements in attentional processes, which in turn were associated with decreased sport injury. Specifically, participants in the mindfulness group experienced improved speed and accuracy of information processing. Perceptual and attentional deficits (e.g., "tunnel vision”) are known antecedents of sport injury. Inability to pay attention to the task and to real time decision making is associated with an increased risk of acute sport injury (Rogers \& Landers, 2005), hence, improved attention might have helped participants identify and react quicker to relevant stimuli, allowing them to function safely within the environment. It may be that mindfulness produced functional changes in attention-related brain areas (Fox, Corbetta, Snyder, Vincent, \& Raichle, 2006), such as reduction of stress-related brain activations, with implications in a variety of cognitive functions such as attention, decision making, and neurocognitive reaction time (Goldin \& Gross, 2010; Perna et al., 2003). Studies have reported that mindfulness practice, where improvements of attention have been observed, 
were associated with changes of the anterior cingulate cortex (Hölzel et al., 2011; Hölzel et al., 2007). Overall, such changes can improve the ability of soccer players to pay attention to task-relevant stimuli during the competition and training and prevent attentional deficits.

Second, participants in the mindfulness group demonstrated decreases in trait anxiety and perceived stress compared to the control group. In addition, changes in trait anxiety and perceived stress were significant mediators of the relationship between the intervention and sport injury, such that decreases in trait anxiety and stress as a result of the intervention were associated with decreases in sport injury. Trait anxiety and perceived stress accounted for $61.5 \%$ and $27.5 \%$ of the total effect of mindfulness on decreased sport injury, respectively. These results are important because according to the stress and athletic injury model, athletes who experience higher levels of stress and anxiety are at greater risk of acute sport injury (Williams \& Andersen, 1998). Interventions that target stress appraisals or the stress response can be useful in reducing the risk of acute sport injury (Brewer \& Redmond, 2017) due to a less intense stress response. Considering that trait anxiety is a relatively stable personality characteristic, the idea of reductions of trait anxiety after a 7-week intervention may seem counterintuitive. However, these findings replicate research showing increased trait mindfulness and reductions in sport-related trait anxiety in recreational runners (De Petrillo, Kaufman, Glass, \& Arnkoff, 2009) and mixed-sport training in collegiate athletes (Glass, Spears, Perskaudas, \& Kaufman, 2018). The present results may suggest that, after a 7-week mindfulness training, the subscales of trait sport anxiety, worry, and concentration disruption decreased in the mindfulness group relative to pretest but not in the control group. Therefore, consistent with the findings of Jain et al. (2007), mindfulness training can help athletes to control the negative concerns about potential negative personal often expressed in ruminative thoughts and catastrophizing. 
Third, participants in the mindfulness group and non-injured athletes reported increases

500

501

502

503

504

505

506

507

508

509

510

511

512

513

514

515

516

517

518

519

520

521

522

523

in state and trait mindfulness. Moreover, mediation analysis suggests that the negative indirect effects of state mindfulness accounted for $16.8 \%$ of the total effects of mindfulness on the decreased risk of sport injury. Its indirect effects were the smallest of all the mediators, but it is possible that state mindfulness may act through its influence on other variables. For example, mindfulness practice may reduce emotional interference during sports training and competition (Ivarsson, Johnson, Andersen, et al., 2015) through increased attention to the current context and decreased continued processing of emotional stimuli (Ortner, Kilner, \& Zelazo, 2007). Increased state mindfulness after mindfulness training may help reduce the detrimental experiences of sport-related anxiety (Scott-Hamilton \& Schutte, 2016) and improve future outcome expectations and attention. In addition, individuals varied significantly their rates of change in state mindfulness after interventions, and these individual trajectories predicted pre-post intervention changes in trait mindfulness and distress (Kiken, Garland, Bluth, Palsson, \& Gaylord, 2015). Other studies have also found that, on average, mindfulness-based interventions increase trait mindfulness, which contributes to psychological health (Carmody, Reed, Kristeller, \& Merriam, 2008; Shapiro, Oman, Thoresen, Plante, \& Flinders, 2008).

Although mindfulness training is not specifically mentioned in the Andersen and Williams model, based on our study we argue that mindfulness training can reduce the stress response by attenuating negative appraisals of potentially stressful athletic situations (e.g., an upcoming competition) and facilitating the use of adaptive forms of coping, such as positive reappraisals (Weinstein et al., 2009). Positive reappraisals refer to the adaptive process through which stressful events are re-construed as benign, useful, and meaningful (Garland, Gaylord, \& Park, 2009). It is an active and meaning-based coping strategy, which often is the first step towards productive reengagement with stressful events (Folkman, 1997). 
524 Mindfulness training can help individuals develop positive reappraisals in the face of stress 525 a cognitive coping style (Garland, Gaylord \& Fredrickson, 2011).

526 Garland et al. (2011), when individuals cognitively appraise situations as threats, they

527 may cope by decentering from this stress appraisal into a mindfulness mode, wherein

528 individuals attend to the dynamic process of consciousness itself rather than its contents. This

529 state of consciousness is "characterized by broadened attention, awareness, and increased 530 cognitive flexibility" (p. 60) that help the individual to disengage and withdraw from the

531 initial appraisal into a momentary state of metacognitive awareness that attenuates semantic

532 evaluations associated with the event. Henceforth, people can reframe their initial negative

533 circumstances as meaningful or even advantageous. With persistent engagement with this

534 metacognitive awareness, a mindful disposition may develop and a coping cognitive style

535 based on cognitive reappraisals may emerge, leading to reduced stress. Further supporting

536 this argument, in the current study, following mindfulness training, trait and state

537 mindfulness improved in the uninjured players compared to the injured players, with large

538 effect sizes suggesting important clinical benefits of the intervention. In addition, changes in

539 state mindfulness mediated the relationship between mindfulness training and soccer injury.

540 Nevertheless, in our study the role of cognitive reappraisals as a coping strategy for stressful

541 situations was not considered; therefore, this variable should be measured in future studies to

542 explore the extent to which it mediates the relationship between mindfulness and injury.

543 The current study has several limitations that should be noted. This study was conducted with

544 male soccer players between 16 and 19 years of age; hence, generalizability to other age and

545 gender groups is not possible. Age differences can be expected as a result of injury history

546 and a trend for older people to receive long-term interventions. Gender differences in the

547 occurrence of sports injuries may affect the effectiveness of the program (Powell \& Barber-

548 Foss, 2000), although this program does not seem to be gender-specific; hence, we suggest 
that this program can be offered to both male and female athletes. In our study, we retrospectively obtained the injury data for the pre-intervention period (past season), by means of an individual interview conducted by a physical therapist and a medical student. Participants' reports might have been inaccurate due to reliance on memory, resulting in underestimation or overestimation of the number of injuries occurred during the previous season. However, to minimize this limitation, in the present study, we used a control group and matched participants on number of previous injuries; this strategy was deemed relevant because it has been reported that previous injuries are strong predictors of both re-injury and new injuries (Hägglund, Waldén, \& Ekstrand, 2006). Due to potential difficulties with the recall of past injuries, in the current study we considered only the injuries that athletes had experienced in the previous season. Nevertheless, major injuries from past seasons may also have an impact on perceived stress and current injuries. Hence, a longer injury history should be considered in future research. Another limitation refers to the operational definition of injury, which led us to disregard those injuries that resulted in fewer than 4 days of loss time for the participants. The instruments chosen to measure mindfulness were not sport-specific. Because a sport-specific mindfulness measure (Thienot et al., 2014) at the time the study, we selected instruments that have been successfully applied in studies in sport (Noetel et al., 2017). In addition, we did not formally record the duration of the home-based mindfulness exercises in the intervention group after the seven formal training sessions. The duration of such engagement component is likely to influence the effectiveness of intervention. Also, although athletes from the same team were allocated to different groups, we did not formally control the possible exchange of information between them. Therefore, the potential for knowledge exchange existed, which may have confounded the results of the intervention. To analyze the indirect effects of the mindfulness program on injuries we used Hayes' (2013) mediation analysis approach and used the pre-test scores of the mediators as covariates. A 
574 stronger approach would be to utilize structural equation models to fully control for

575 measurement errors; however, this approach has larger sample size requirements.

576 Furthermore, the model proposed suggested the existence of a suppression effect caused by

577 attention. Therefore, further studies should be conducted to clarify the role of

578 attention.Finally, assessing the long-term benefits of this intervention can also be useful to

579 decide on its cost effectiveness, that is, whether the proposed cost of prevention is lower than 580 the costs associated with sports injuries. Hence, follow-up studies are needed. The results of

581 this study suggest that a mindfulness training program can reduce the occurrence of acute

582 sport injuries through improvements in attention, trait anxiety, perceived stress and state

583 mindfulness. To understand the value of these results, we recall Benjamin Franklin's

584 statement: "An ounce of prevention is worth a pound of cure." Given that mindfulness

585 exercises can be conducted prior to, during, or after physical practice and because athletes

586 and coaches were satisfied with the effect of mindfulness training and were willing to use

587 such a program in the future, it is suggested that athletes should incorporate these exercises

588 into their practice.

589 Acknowledgements. The authors would like to thank the all the soccer players who

590 participated in this study and coaches who collaborated to conduct this study. 
591

592

593

594

595

596

597

598

599

600

601

602

603

604

605

606

607

608

609

610

611

612

613

614

615

616

\section{References}

Andrews, G., \& Slade, T. (2001). Interpreting scores on the Kessler Psychological Distress Scale (K10). Australian and New Zealand Journal of Public Health, 25, 494-497. doi:10.1111/j.1467-842X.2001.tb00310.x

Barkley, R. A. (1991). The ecological validity of laboratory and analogue assessment methods of ADHD symptoms. Journal of Abnormal Child Psychology, 19, 149-178.

Bates, M. E., \& Lemay, E. P. (2004). The d2 Test of attention: construct validity and extensions in scoring techniques. Journal of the International Neuropsychological Society, 10, 392-400. doi:10.1017/S135561770410307X.

Bergomi, C., Tschacher, W., \& Kupper, Z. (2014). Konstruktion und erste Validierung eines Fragebogens zur umfassenden Erfassung von Achtsamkeit [Construction and first validation of the Comprehensive Inventory of Mindfulness Experiences]. Diagnostica. doi:10.1026/0012-1924/a000109.

Brewer, B. W., \& Redmond, C. (2017). Psychology of Sport Injury (First ed.). United State of America: Human Kinetics.

Brown, K. W., \& Ryan, R. M. (2003). The benefits of being present: mindfulness and its role in psychological well-being. Journal of Personality and Social Psychology, 84, 822-828. doi:10.1037/0022-3514.84.4.822

Caglar, E., \& Koruc, Z. (2006). Reliability and validity of d2 test of attention for athletes. Hacettepe Journal of Sport Sciences, 17, 58-80.

Carmody, J., Reed, G., Kristeller, J., \& Merriam, P. (2008). Mindfulness, spirituality, and healthrelated symptoms. Journal of Psychosomatic Research, 64, 393-403. doi:10.1016/j.jpsychores.2007.06.015

De Petrillo, L. A., Kaufman, K. A., Glass, C. R., \& Arnkoff, D. B. (2009). Mindfulness for longdistance runners: An open trial using Mindful Sport Performance Enhancement (MSPE). Journal of Clinical Sport Psychology, 3, 357-376. doi:10.1123/jcsp.3.4.357 
617

618

619

620

621

622

623

624

625

626

627

628

629

630

631

632

633

634

635

636

637

638

639

640

641

642

Drew, M. K., Raysmith, B. P., \& Charlton, P. C. (2017). Injuries impair the chance of successful performance by sportspeople: a systematic review. British Journal of Sports Medicine 12091214. doi:10.1136/bjsports-2016-096731.

Edvardsson, A., Ivarsson, A., \& Johnson, U. (2012). Is a cognitive-behavioural biofeedback intervention useful to reduce injury risk in junior football players? Journal of Sports Science and Medicine, 11, 331.

Ekstrand, J. (2013). Keeping your top players on the pitch: the key to football medicine at a professional level. British Journal of Sports Medicine 47, 723-724. doi:10.1136/bjsports2013-092771.

Folkman, S. (1997). Positive psychological states and coping with severe stress. Social Science \& Medicine, 45, 1207-1221.

Fox, M. D., Corbetta, M., Snyder, A. Z., Vincent, J. L., \& Raichle, M. E. (2006). Spontaneous neuronal activity distinguishes human dorsal and ventral attention systems. Proceedings of the National Academy of Sciences, 103, 10046-10051. doi:10.1073/pnas.0604187103.

Fuller, C. W., Ekstrand, J., Junge, A., Andersen, T. E., Bahr, R., Dvorak, J., . . Meeuwisse, W. H. (2006). Consensus statement on injury definitions and data collection procedures in studies of football (soccer) injuries. Scandinavian Journal of Medicine \& Science in Sports, 16, 83-92. doi:10.1097/00042752-200603000-00003

Gardner, F. L., \& Moore, Z. E. (2007). The psychology of enhancing human performance: The mindfulness-acceptance-commitment (MAC) approach (First ed.). New York: Springer Publishing Company.

Garland, E., Gaylord, S., \& Park, J. (2009). The role of mindfulness in positive reappraisal. Explore, 5, 37-44. doi:10.1016/j.explore.2008.10.001

Garland, E. L., Gaylord, S. A., \& Fredrickson, B. L. (2011). Positive reappraisal mediates the stressreductive effects of mindfulness: An upward spiral process. Mindfulness, 2, 59-67. doi:10.1007/s12671-011-0043-8 
Glass, C. R., Spears, C. A., Perskaudas, R., \& Kaufman, K. A. (2018). Mindful sport performance enhancement: Randomized controlled trial of a mental training program with collegiate athletes. Journal of Clinical Sport Psychology, 1-34. doi:10.1123/jcsp.2017-0044

Goldin, P. R., \& Gross, J. J. (2010). Effects of mindfulness-based stress reduction (MBSR) on emotion regulation in social anxiety disorder. Emotion, 10, 83. doi:10.1037/a0018441.

Hägglund, M. (2007). Epidemiology and Prevention of Football Injuries. (Doctoral dissertation), Linköpings Universitet.

Hägglund, M., Waldén, M., \& Ekstrand, J. (2006). Previous injury as a risk factor for injury in elite football: a prospective study over two consecutive seasons. British Journal of Sports Medicine 40, 767-772. doi:10.1136/bjsm.2006.026609.

Hayes, A. F. (2013). Introduction to mediation, moderation, and conditional process analysis: A regression-based approach (Second ed.). New York: Guilford Press.

Hayes, S. C., Strosahl, K. D., \& Wilson, K. G. (2012). Acceptance and commitment therapy (Second ed.). New York: Guilford Press

Hölzel, B. K., Lazar, S. W., Gard, T., Schuman-Olivier, Z., Vago, D. R., \& Ott, U. (2011). How does mindfulness meditation work? Proposing mechanisms of action from a conceptual and neural perspective. Perspectives on Psychological Science, 6, 537-559. doi:10.1177/1745691611419671.

Hölzel, B. K., Ott, U., Hempel, H., Hackl, A., Wolf, K., Stark, R., \& Vaitl, D. (2007). Differential engagement of anterior cingulate and adjacent medial frontal cortex in adept meditators and non-meditators. Neuroscience Letters, 421, 16-21. doi:10.1016/j.neulet.2007.04.074.

Ivarsson, A., Johnson, U., Andersen, M. B., Fallby, J., \& Altemyr, M. (2015). It pays to pay attention: A mindfulness-based program for injury prevention with soccer players. Journal of Applied Sport Psychology, 27, 319-334. doi:10.1080/10413200.2015.1008072.

Ivarsson, A., Johnson, U., Andersen, M. B., Tranaeus, U., Stenling, A., \& Lindwall, M. (2017). Psychosocial factors and sport injuries: meta-analyses for prediction and prevention. Sports Medicine, 47, 353-365. doi:10.1007/s40279-016-0578-x. 
670 Ivarsson, A., Johnson, U., \& Edvardsson, A. (2015). Psychologically based programs for injury prevention in football: a meta-analysis. Paper presented at the 8th World Congress on Science and Football, Copenhagen, Denmark, 20-23 May, 2015.

Johnson, U., Ekengren, J., \& Andersen, M. B. (2005). Injury prevention in Sweden: Helping soccer players at risk. Journal of Sport and Exercise Psychology, 27, 32-38. doi:10.1123/jsep.27.1.32.

Kessler, R. C., Andrews, G., Colpe, L. J., Hiripi, E., Mroczek, D. K., Normand, S.-L., . . Zaslavsky, A. M. (2002). Short screening scales to monitor population prevalences and trends in nonspecific psychological distress. Psychological Medicine, 32, 959-976.

Kiken, L. G., Garland, E. L., Bluth, K., Palsson, O. S., \& Gaylord, S. A. (2015). From a state to a trait: Trajectories of state mindfulness in meditation during intervention predict changes in trait mindfulness. Personality and Individual differences, 81, 41-46. doi:10.1016/j.paid.2014.12.044

Lau, M. A., Bishop, S. R., Segal, Z. V., Buis, T., Anderson, N. D., Carlson, L., . . Devins, G. (2006). The Toronto mindfulness scale: Development and validation. Journal of Clinical Psychology, 62, 1445-1467. doi:10.1002/jclp.20326.

MacKinnon, D. P., Krull, J. L., \& Lockwood, C. M. (2000). Equivalence of the mediation, confounding and suppression effect. Prevention Science, 1, 173-181. doi.org/10.1023/A:1026595011371

Noetel, M., Ciarrochi, J., Van Zanden, B., \& Lonsdale, C. (2017). Mindfulness and acceptance approaches to sporting performance enhancement: A systematic review. International Review of Sport and Exercise Psychology, 1, 1-37. doi:10.1080/1750984X.2017.1387803

Ortner, C. N., Kilner, S. J., \& Zelazo, P. D. (2007). Mindfulness meditation and reduced emotional interference on a cognitive task. Motivation and Emotion, 31, 271-283. doi:10.1007/s11031007-9076-7

Perna, F. M., Antoni, M. H., Baum, A., Gordon, P., \& Schneiderman, N. (2003). Cognitive behavioral stress management effects on injury and illness among competitive athletes: a randomized 

clinical trial. Annals of Behavioral Medicine, 25, 66-73. doi:10.1207/S15324796ABM2501_09.

Pfirrmann, D., Herbst, M., Ingelfinger, P., Simon, P., \& Tug, S. (2016). Analysis of injury incidences in male professional adult and elite youth soccer players: a systematic review. Journal of Athletic Training, 51, 410-424. doi:10.4085/1062-6050-51.6.03.

Plemmons, M. G. (2015). Evaluation of the Effectiveness of the Mindfulness-acceptance-commitment (MAC) Approach in Recreational Golfers. (Master of arts), Appalachian State University Boone, NC, USA.

Powell, J. W., \& Barber-Foss, K. D. (2000). Sex-related injury patterns among selected high school sports. The American Journal of Sports Medicine, 28, 385-391. doi:10.1177/03635465000280031801.

Putukian, M. (2016). The psychological response to injury in student athletes: a narrative review with a focus on mental health. British Journal of Sports Medicine 50, 145-148. doi:10.1136/bjsports-2015-095586.

Rogers, T. J., \& Landers, D. M. (2005). Mediating effects of peripheral vision in the life event stress/athletic injury relationship. Journal of Sport and Exercise Psychology, 27, 271-288. doi:10.1123/jsep.27.3.271

Scott-Hamilton, J., \& Schutte, N. S. (2016). The role of adherence in the effects of a mindfulness intervention for competitive athletes: Changes in mindfulness, flow, pessimism, and anxiety. Journal of Clinical Sport Psychology, 10, 99-117.

Segal, Z. V., Teasdale, J. D., Williams, J. M., \& Gemar, M. C. (2002). The mindfulness-based cognitive therapy adherence scale: Inter-rater reliability, adherence to protocol and treatment distinctiveness. Clinical Psychology \& Psychotherapy, 9, 131-138. doi:10.1002/cpp.320.

Shapiro, S. L., Oman, D., Thoresen, C. E., Plante, T. G., \& Flinders, T. (2008). Cultivating mindfulness: effects on well-being. Journal of Clinical Psychology, 64, 840-862. doi:10.1002/jclp.20491 
Smith, R. E., Smoll, F. L., Cumming, S. P., \& Grossbard, J. R. (2006). Measurement of multidimensional sport performance anxiety in children and adults: The Sport Anxiety Scale2. Journal of Sport and Exercise Psychology, 28, 479-501. doi:10.1123/jsep.28.4.479

Stubbe, J. H., van Beijsterveldt, A.-M. M., van der Knaap, S., Stege, J., Verhagen, E. A., Van Mechelen, W., \& Backx, F. J. (2015). Injuries in professional male soccer players in the Netherlands: a prospective cohort study. Journal of Athletic Training, 50, 211-216. doi:10.4085/1062-6050-49.3.64.

Thienot, E., Jackson, B., Dimmock, J., Grove, J. R., Bernier, M., \& Fournier, J. F. (2014). Development and preliminary validation of the mindfulness inventory for sport. Psychology of Sport and Exercise, 15, 72-80. doi:10.1016/j.psychsport.2013.10.003

Tranaeus, U., Johnson, U., Engström, B., Skillgate, E., \& Werner, S. (2015). A psychological injury prevention group intervention in Swedish floorball. Knee Surgery, Sports Traumatology, Arthroscopy, 23, 3414-3420. doi:10.1007/s00167-014-3133-z.

Weinstein, N., Brown, K. W., \& Ryan, R. M. (2009). A multi-method examination of the effects of mindfulness on stress attribution, coping, and emotional well-being. Journal of Research in Personality, 43, 374-385. doi:10.1016/j.jrp.2008.12.008.

Williams, J. M., \& Andersen, M. B. (1998). Psychosocial antecedents of sport injury: Review and critique of the stress and injury model'. Journal of Applied Sport Psychology, 10, 5-25. doi:10.1080/10413209808406375. 
744 Table 1. Repeated ANOVA results for comparison of psychological factors between the control and mindfulness groups.

\begin{tabular}{|c|c|c|c|c|c|c|c|c|c|c|c|c|c|}
\hline \multirow[t]{2}{*}{ Variables } & \multirow[t]{2}{*}{ Time } & \multirow[t]{2}{*}{$\begin{array}{l}\text { Mindfulness } \\
\text { Group }(n=81)\end{array}$} & \multirow[t]{2}{*}{$\begin{array}{l}\text { Control Group } \\
(\mathrm{n}=79)\end{array}$} & \multirow{2}{*}{$\begin{array}{c}\text { Between Group } \\
\text { Difference } \\
\text { Mean }(95 \% \mathrm{CI})\end{array}$} & \multicolumn{3}{|c|}{ Time effects } & \multicolumn{3}{|c|}{ Group effects } & \multicolumn{3}{|c|}{$\begin{array}{l}\text { Time } \times \text { group } \\
\text { interaction }\end{array}$} \\
\hline & & & & & $\begin{array}{l}F_{(I,} \\
158)\end{array}$ & $p$ & $\eta^{2}$ & $F_{(1,158}$ & $p$ & $\eta^{2}$ & $F_{(1,158}$ & $p$ & $\eta^{2}$ \\
\hline \multirow{2}{*}{$\begin{array}{c}\text { Trait } \\
\text { mindfulness }\end{array}$} & Before & $134.52 \pm 12.63$ & $135.32 \pm 12.56$ & $-0.81(-4.6 ; 3.1)$ & 10.2 & 0.002 & 0.0 & 0.82 & 0.3 & 0.00 & 7.52 & 0.007 & 0.0 \\
\hline & after & $139.94 \pm 13.31$ & $134.71 \pm 13.28$ & $3.21(-0.8 ; 7.4)$ & 1 & $*$ & 6 & & 7 & 5 & & $*$ & 4 \\
\hline \multirow{2}{*}{$\begin{array}{c}\text { State } \\
\text { mindfulness }\end{array}$} & Before & $24.15 \pm 5.36$ & $24.42 \pm 6.41$ & $-031(-2.1 ; 1.5)$ & 6.43 & $0.01^{*}$ & 0.0 & 1.10 & 0.3 & 0.01 & 9.36 & 0.003 & 0.0 \\
\hline & after & $27.03 \pm 5.73$ & $24.32 \pm 6.18$ & $2.00(0.2 ; 3.8)$ & & & 4 & & 0 & & & $*$ & 5 \\
\hline \multirow{2}{*}{$\begin{array}{l}\text { Trait sport } \\
\text { Anxiety }\end{array}$} & Before & $35.03 \pm 5.81$ & $35.36 \pm 5.83$ & $-0.53(-1.5 ; 0.2)$ & 27.2 & 0.001 & 0.1 & 5.79 & 0.0 & 0.30 & 15.23 & 0.001 & 0.0 \\
\hline & after & $31.15 \pm 6.43$ & $34.79 \pm 5.93$ & $-1.10(-1.6 ;-0.1)$ & 2 & $*$ & 4 & & 2 & & & $*$ & 8 \\
\hline \multirow[t]{2}{*}{ Stress } & Before & $10.84 \pm 3.98$ & $10.97 \pm 4.36$ & $0.09(-1.4 ; 1.1)$ & 26.7 & 0.001 & 0.1 & 1.88 & 0.1 & 0.01 & 14.34 & 0.001 & 0.0 \\
\hline & after & $9.15 \pm 3.83$ & $10.71 \pm 4.49$ & $-1.42(-2.9 ;-0.4)$ & 8 & $*$ & 4 & & 7 & & & $*$ & 8 \\
\hline \multirow{2}{*}{$\begin{array}{l}\text { Attention } \\
\text { process }\end{array}$} & Before & $3.28 \pm 1.78$ & $3.29 \pm 1.82$ & $-0.01(-0.6 ; 0.5)$ & 26.1 & 0.001 & 0.1 & 1.44 & 0.1 & 0.01 & 11.52 & 0.001 & 0.0 \\
\hline & after & $4.14 \pm 1.86$ & $3.52 \pm 1.91$ & $0.62(0.07 ; 1.2)$ & 3 & $*$ & 4 & & 8 & & & $*$ & 7 \\
\hline
\end{tabular}

745 Note. ${ }^{*}$ significant level at $\leq .01$. 
747 Table 2. Injury characteristics of the control and the mindfulness groups.

\begin{tabular}{|c|c|c|c|c|}
\hline Variables & $\begin{array}{l}\text { Mindfulness Group } \\
(\mathrm{n}=81)\end{array}$ & $\begin{array}{l}\text { Control Group } \\
(\mathrm{n}=79)\end{array}$ & $\mathrm{T}$ or $\chi^{2}$ & p-value \\
\hline \multicolumn{5}{|c|}{ Number of athlete-exposure } \\
\hline Total & 5942 & 5887 & & \\
\hline Games & 1351 & 1403 & & \\
\hline Practices & 4591 & 4485 & & \\
\hline \multicolumn{5}{|c|}{ Injuries, $n^{\mp}$} \\
\hline Total & 22 & 36 & 5.09 & 0.02 \\
\hline Game & 14 & 22 & 2.34 & 0.09 \\
\hline Practice & 8 & 14 & 1.86 & 0.12 \\
\hline \multicolumn{5}{|c|}{ Days lost to injury, $n(\text { mean } \pm S D)^{\dagger}$} \\
\hline & $218(9.88 \pm 8.56)$ & $516(14.32 \pm 12.28)$ & 4.1 & 0.03 \\
\hline \multicolumn{5}{|c|}{ Injury intensity, $n(\%)^{+}$} \\
\hline Mild & $14(63.47)$ & $18(50)$ & \multirow{3}{*}{1.34} & \multirow{3}{*}{0.48} \\
\hline Moderate & $6(27.52)$ & $15(42)$ & & \\
\hline Sever & $2(9)$ & $3(8)$ & & \\
\hline \multicolumn{5}{|c|}{ Incidence rate per 1000 athlete-exposure* } \\
\hline Total & 3.67 & 6.12 & \multirow{3}{*}{2.76} & \multirow{3}{*}{0.03} \\
\hline Game & 10.43 & 15.68 & & \\
\hline Practice & 1.72 & 3.13 & & \\
\hline
\end{tabular}

Note. ${ }^{\dagger}$ independent $t$-test analysis; ${ }^{\star}$ chi-square analysis 


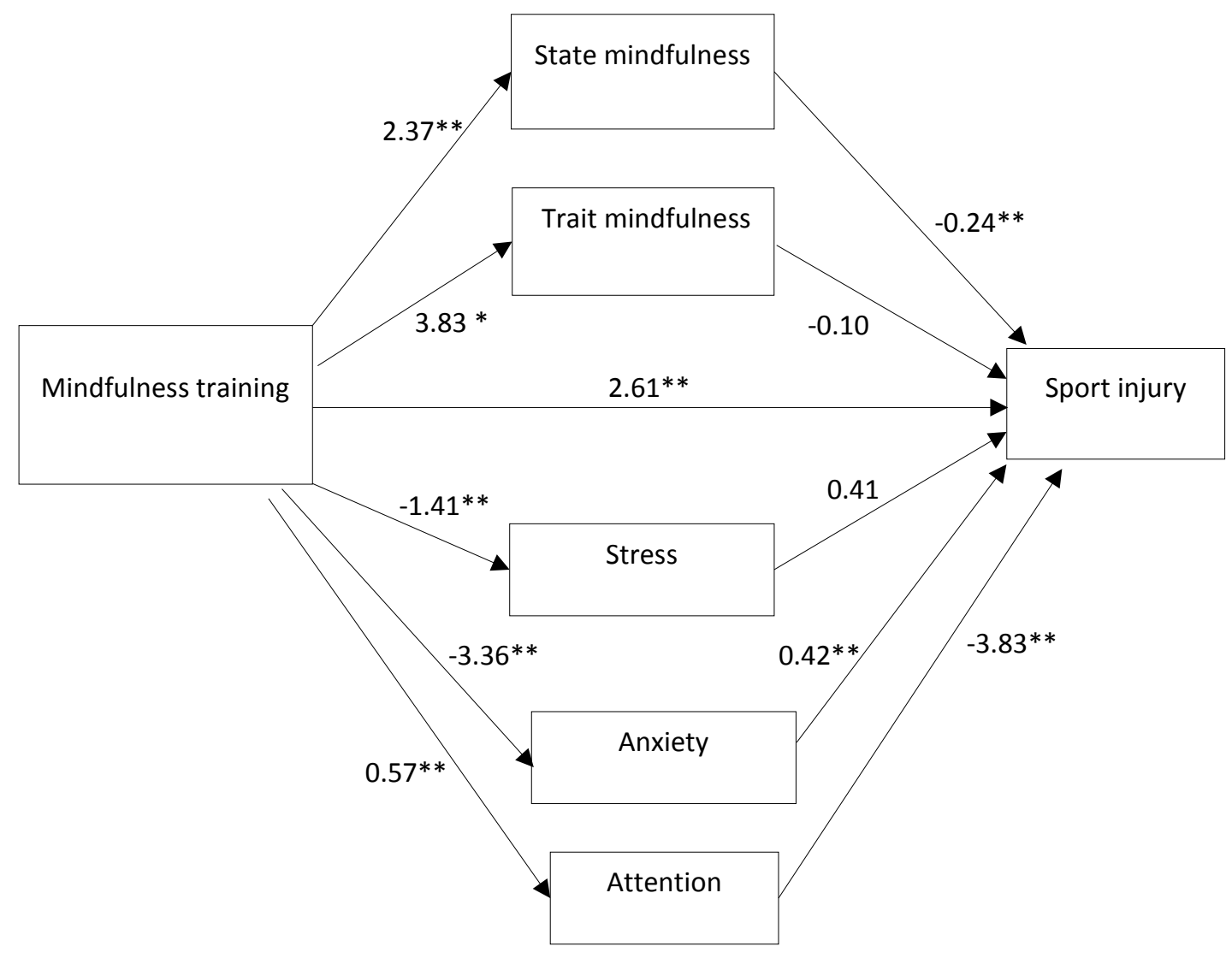

753

754 Figure 1. Path model of changes in psychological variables as mediators of the effects of

755 mindfulness training on acute sport injury. Note: Sport injury was coded such that $0=$ non-

756 injured and $1=$ injured. Group assignment was coded such that $0=$ control group and $1=$

757 mindfulness training. 5000 Bootstrapping samples from the original data set were generated

758 by random sampling. Unstandardized coefficients are presented. $* \mathrm{p} \leq .05, * * \mathrm{p}<.01, * * * \mathrm{p}$

$759<.001$.

760 